\title{
Central Thalamic Contributions to Arousal Regulation and Neurological Disorders of Consciousness
}

\author{
Nicholas D. Sahiff \\ Department of Neurology and Neuroscience, Weill Cornell Medical College,
} New York, New York, USA

\begin{abstract}
This review focuses on the contributions of the central thalamus to normal mechanisms of arousal regulation and to neurological disorders of consciousness. Forebrain arousal is regulated by ascending influences from brainstem/basal forebrain neuronal populations ("arousal systems") and control signals descending from frontal cortical systems. These subcortical and cortical systems have converging projections to the central thalamus that emphasize their role in maintaining organized behavior during wakefulness. Central thalamic neurons appear to be specialized both anatomically and physiologically to support distributed network activity that maintains neuronal firing patterns across long-range cortico-cortical pathways and within cortico-striatopallidalthalamocortical loop connections. Recruitment of central thalamic neurons occurs in response to increasing cognitive demand, stress, fatigue, and other perturbations that reduce behavioral performance. In addition, the central thalamus receives projections from brainstem pathways evolved to rapidly generate brief shifts of arousal associated with the appearance of salient stimuli across different sensory modalities. Through activation of the central thalamus, neurons across the cerebral cortex and striatum can be depolarized and their activity patterns selectively gated by descending or ascending signals related to premotor attention and alerting stimuli. Direct injury to the central thalamus or prominent deafferentation of these neurons as a result of complex, multifocal, brain insults are both associated with severe impairment of forebrain functional integration and arousal regulation. Interventions targeting neurons within the central thalamus may lead to rational therapeutic approaches to the treatment of impaired arousal regulation following nonprogressive brain injuries. A model accounting for present therapeutic strategies is proposed.
\end{abstract}

Key words: intralaminar nuclei; minimally conscious state; calbindin; deep brain stimulation

\section{The Central Thalamus and Arousal Regulation}

Many neurons within the central thalamus (defined here as the anterior and posterior intralaminar nuclei and the paralaminar portions of related thalamic association nuclei - median dorsalis, ventral anterior, ventral lateral, and inferior pulvinar) share specific anatomical and physiological specializations that support their key role in the general functions of sustained attention, working memory, and motor preparation. These neuronal populations are interposed between

Address for correspondence: Nicholas D. Schiff, Associate Professor of Neurology and Neuroscience, Director, Laboratory of Cognitive Neuromodulation, Department of Neurology and Neuroscience, Weill Medical College of Cornell University, New York, NY 10065. Voice: +1-212-7462372; fax: +1-212-746-8532.

nds2001@med.cornell.edu brainstem/basal forebrain "arousal systems" that control overall levels of thalamic and cortical activity ${ }^{1,2}$ and supervisory frontal systems ${ }^{3}$ that organize both premotor shifts of attention ${ }^{4,5}$ and adjustments of vigilance level or alertness. ${ }^{6,7}$ In parallel, individual neurons within these cellular aggregates are targets of a variety of dedicated brainstem sensory relays that have evolved to quickly capture attention and redirect behavior (FIG. 1). ${ }^{8,9}$ Collectively, these anatomical specializations suggest that many neurons within the central thalamus may serve a general purpose function supporting large-scale cerebral dynamics associated with goal-directed behaviors and consciousness. In this review, this general function is identified as arousal regulation and the central thalamus is viewed as containing collections of cells that are recruited either 1) as part of broad gain control systems that shift and hold levels of activation across large transiently established networks or 2) by transient activation of these central thalamic neurons that may be used to bias activity within 


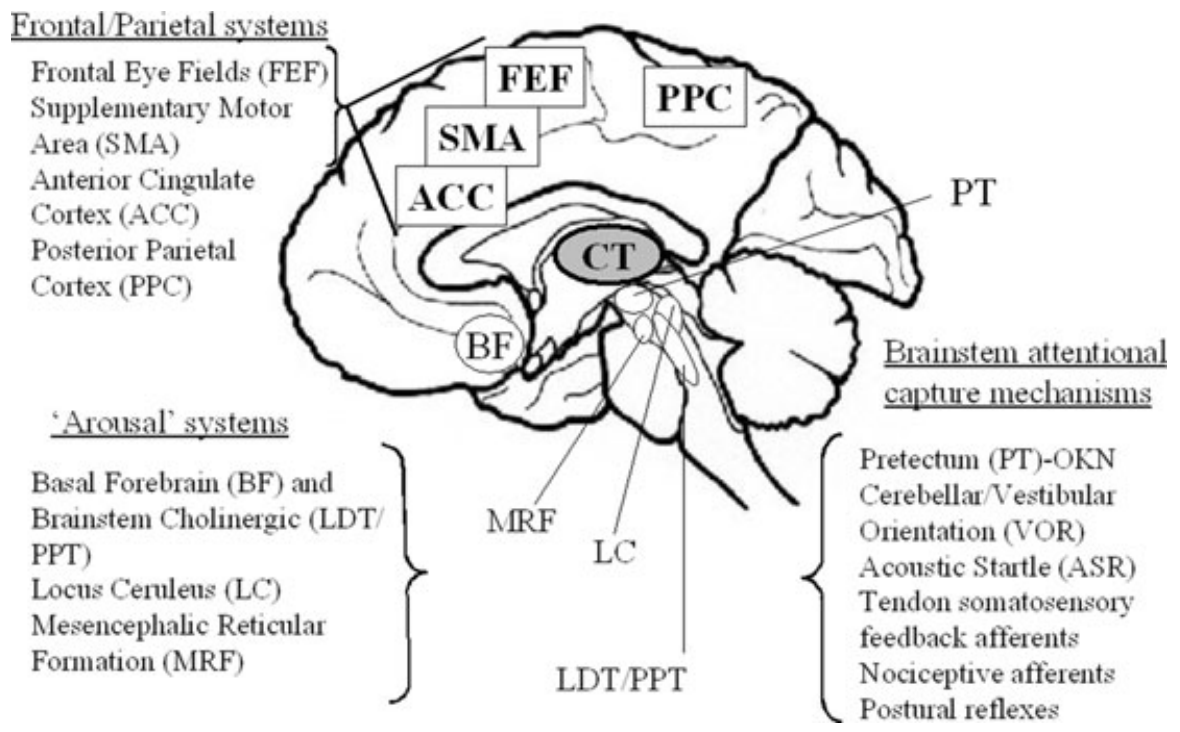

FIGURE 1. Central thalamus (CT) and arousal regulation: connections of the central thalamus. The connections of the central thalamus support its role in arousal regulation mechanisms that adjust activity levels over large-scale networks during behavior. The central thalamus has strong reciprocal connections with several subregions of the frontal cortex engaged in planning and execution of movement, including the frontal eye fields (FEF), supplementary motor area (SMA), anterior cingulate cortex (ACC), and more posterior cortical association areas that support polysensory integration, including posterior parietal cortex (PPC). Bottom-up connections to the central thalamus include projections from the brainstem arousal systems, including cholinergic fibers from the lateral dorsal tegmental nucleus (LDT) and pedunculopontine nucleus (PPT), noradrenergic afferents from locus ceruleus (LC), and glutamatergic afferents from the mesencephalic reticular formation (MRF). Parallel inputs to the central thalamus arise from a variety of brainstem circuits that redirect attention to events in the sensory periphery (see text for further discussion).

and across the hemispheres as required during ongoing behavior.

Historically, neurons within the central thalamus (primarily the intralaminar and paralaminar nuclear groups that produced broad "recruiting responses" in the electroencephalogram (EEG) with low frequency electrical stimulation ${ }^{10}$ became identified with a primary role in maintaining wakefulness. ${ }^{11}$ The classical experimental studies of Moruzzi and Magoun ${ }^{11}$ suggested that the intralaminar thalamic neurons were part of an ascending pathway that originated in the tegmental mesencephalon (midbrain reticular formation [MRF]). Electrical stimulation of the MRF and central thalamus in these studies desynchronized the slow large-amplitude waves of EEG activity induced in anesthetized cats. Confirmation of this anatomical pathway from the MRF to the central thalamus (specifically, anterior intralaminar nuclei/median dorsalis) was later provided by detailed electroanatomical studies in the cat. Steriade and Glenn ${ }^{12}$ recorded extracellularly from histologically identified neurons in the largest cell populations in the anterior intralaminar nu- clei (central lateral [CL] and paracentralis nuclei [Pc]) of the cat thalamus and demonstrated that these neurons could be antidromically activated by electrically stimulating association regions of the cerebral cortex and the caudate nucleus of the striatum or synaptically driven by stimulation of the MRF (after lesioning of all rostral pontine projections to the thalamus). In related studies, simultaneous extracellular recordings from cell bodies in MRF and the anterior intralaminar group (CL, Pc) during spontaneous sleep-wake cycles showed a strong correlation of tonic excitation of MRF neurons and excitability of intralaminar neurons projecting to the cortex during wakefulness. ${ }^{13}$ Further characterization of these CL neurons demonstrated that they showed characteristic firing patterns during wakefulness. ${ }^{14}$ These neurons generated spike bursts at $800-1000 \mathrm{~Hz}$ at a firing rate in the $20-40 \mathrm{~Hz}$ frequency range during wakefulness and rapid eye movement (REM) sleep. Intracellular recordings from the same cells revealed that depolarizing the resting membrane potential triggered fast oscillations $(20-80 \mathrm{~Hz})$ of the short high-frequency $(800-1000 \mathrm{~Hz})$ spike bursts. 
These findings can be compared to human studies that show increased high frequency oscillations arise in the EEG (or magnetoencephalogram) signal from the cerebral cortex during wakefulness and REM sleep that suggest origins in thalamocortical system. ${ }^{15-17} \mathrm{Al}-$ though MRF and CL neurons show clear increases in firing during transitions to wakefulness, ${ }^{13}$ this shift in firing pattern lags a marked increase in brainstem cholinergic neuron firing rates that appear to drive the transition to wakefulness, ${ }^{18}$ most likely in conjunction with activity shifts in several other brainstem, basal forebrain, and hypothalamic populations. ${ }^{19-21}$ Thus, these central thalamic nuclei may play an essential role in supporting the state changes of corticothalamic systems that underlie sleep-wake cycles ${ }^{18,22}$ but are no longer considered to have a primary role in driving the onset of wakefulness - a role now assigned to collections of nuclei in the brainstem and basal forebrain. ${ }^{2,19,23}$

\section{Connections of Central Thalamus with Brainstem and Basal Forebrain Arousal Systems}

The central thalamus receives broad innervation from both the brainstem and basal forebrain arousal systems. ${ }^{9,23,24}$ Ascending inputs to the anterior intralaminar nuclei (CL, Pc) and adjacent paralaminar regions of thalamic association nuclei make them particularly well positioned to play a role in arousal regulation. These regions receive the heaviest thalamic innervation from brainstem and basal forebrain cholinergic neuronal populations ${ }^{1,24}$ and heavy innervation from noradrenergic afferents from the locus ceruleus and serotoninergic afferents from the medial raphe. ${ }^{9,25,26}$ The brainstem and basal forebrain both provide dual cholinergic inputs to the thalamus that activate neurons within the anterior intralaminar region: 1) Inputs arise from both the basal forebrain and brainstem cholinergic groups to the nucleus reticularis (NRT) that hyperpolarize these neurons acting through muscarinic receptors. NRT neurons provide a gabaergic inhibitory influence on the cortically projecting relay neurons in the anterior intralaminar nuclei; 2) inputs to neurons within anterior intralaminar regions that exert direct depolarizing effects acting through nicotinic receptors. ${ }^{1,27}$ In addition to the cholinergic and monoaminergic systems, there are at least two important sources of inputs arising from the glutamatergic components of the arousal system, the parabrachical nucleus and the monosynaptic excitatory pathway from the MRF discussed above. ${ }^{12,19}$

\section{Role of the Central Thalamus in Arousal Regulation within the Wakeful State}

Human imaging studies reveal that selective activation of central thalamus occurs both during tasks requiring short-term shifts of attention (hundreds of milliseconds to seconds ${ }^{28-30}$ ) and during tasks placing sustained demands of high vigilance over extended time periods of hours. ${ }^{31}$ Kinomura et al..$^{28}$ identified strong activation of both the rostral (CL, Pc) and caudal intralaminar nuclei (centromedian-parafasicularis complex, Cm-Pf) during the short-term shifting of attention component of a forewarned reaction time task ("phasic alerting") using functional positron emission tomography. In addition to activation of these thalamic regions, the MRF showed a selective activation consistent with earlier physiological studies identifying this pathway of ascending activation. ${ }^{11,12}$ Paus et al. ${ }^{31}$ studied normal volunteers carrying out a long-term monitoring task (approximately $60 \mathrm{~min}$ ) and demonstrated that vigilance decrements over time correlated with decreased blood flow in the medial thalamus (placed by stereotaxic coordinates in the central lateral and medial dorsalis nuclei $\left.{ }^{31,32}\right)$. Regression analysis showed that this reduced thalamic blood flow significantly covaried with parallel decreases in the anterior cingulate cortex (ACC) and ponto-mesencephalon. The ACC has reciprocal connections with the anterior intralaminar nuclei ${ }^{33}$ and provides wide layer I projections to the prefrontal cortex suggesting it may transfer activation widely throughout the prefrontal cortex. ${ }^{34}$ The ACC is recruited by a diverse range of cognitive demands ${ }^{35}$ and is activated with increasing cognitive load across wide varieties of tasks. ${ }^{36}$ The ACC may drive CL and other central thalamic regions to recruit broader cortical activation in response to demands on effort. Evidence from rat studies that neurons within the ACC monitor and regulate effort allocation, ${ }^{37}$ the covariation of central thalamus with ACC deactivation during vigilance decrements, ${ }^{31}$ and known anatomical connections with $\mathrm{CL}^{38}$ are consistent with this mechanism. CL also has strong reciprocal projections with the supplementary motor area (SMA) of the medial frontal cortex that is also shown to activate during forewarned reaction time tasks ${ }^{30}$ and grade activation with rapidity of response. ${ }^{29}$ Combined use of functional MRI techniques (fMRI) and EEG techniques demonstrate that thalamus and anterior cingulate show selective activation during the appearance of a characteristic direct current (DC) electrical potential associated with anticipatory attention supporting this general model. ${ }^{30}$

Electrophysiological recordings from the central thalamus in alert monkeys performing forewarned 
reaction time tasks demonstrate that a subpopulation of neurons distributed throughout the central thalamus show significant modulation of firing rates during the delay period of the task. ${ }^{39,40}$ The increased firing rate of these neurons is correlated with increased power in the $30-80 \mathrm{~Hz}$ frequency range of the local field potentials, suggesting that synaptic inputs driving the neurons participate in broad patterns of network activation. Other physiological studies have shown that short-term alterations of attentional state are identified with increases in $20-80 \mathrm{~Hz}$ spectral content of the local field potential in several cortical regions recorded in alert nonhuman primates. ${ }^{41-43}$ Episodic modulation of high frequency oscillatory activity in the $20-80 \mathrm{~Hz}$ range has been observed during attentional control, ${ }^{41}$ motor preparation, ${ }^{42}$ and working memory. ${ }^{43}$ Such shifts of neuronal firing rates within the central thalamus may play an important role in facilitating interareal cortico-cortical interactions that are crucial to the performance of many behavioral tasks. ${ }^{28,31,44-47}$ These brief increases of power in $20-80 \mathrm{~Hz}$ range can be compared with the general increase of power in this frequency range seen in cortical recordings that correlate with the shift in firing rates during depolarization of central thalamic neurons during wakefulness and REM states discussed above. ${ }^{14,48}$ Similar findings of increased power in the $35-50 \mathrm{~Hz}$ frequency range have been shown to arise with direct electrical stimulation of the brainstem cholinergic populations and abolished by systemic scopolamine injection. ${ }^{49}$

Taken together, these physiological observations suggest that central thalamic neurons participate in arousal regulation (as operationalized in forewarned reaction time tasks $)^{6}$ as part of a broad network response seen during wakeful states with depolarization of cells producing increased firing in the $20-80 \mathrm{~Hz}$ frequency band, and variations of intensity and temporal shaping of this activity occurring in association with specific task demands. The adjustment of this distributed activation pattern across cerebral structures is likely controlled jointly by tonic levels of brainstem arousal system firing rates and top-down signals from frontal regions involved in monitoring demands on effort and vigilance.

\section{Contribution of Connections of Central Thalamus with the Striatum}

An important anatomical specialization of the intralaminar components of the central thalamus that supports an overall role in shifting levels of activity across broad cerebral networks is their strong efference to the striatum. The anterior and posterior intralam- inar groups are densely populated with neurons with wide projections to striatum as well as cortical targets and collectively provide the largest thalamic efference to the striatum..$^{50-52}$ Anatomical studies demonstrate that individual intralaminar nuclei project to selected groups of cortical and subcortical targets. ${ }^{52,53-55}$ The rostral intralaminar nuclei $(\mathrm{CL}, \mathrm{Pc}$, and central medial $[\mathrm{CeM}])$ tend to project to prefrontal, premotor, posterior parietal, and primary sensory areas and provide a diffuse innervation of the striatum $;^{52}$ the caudal intralaminar nuclei represented by the centromedianparafasicularis complex (Cm-Pf) contain different cell populations with $\mathrm{Cm}$ forming very spatially restricted connections with the striatum $(\mathrm{Cm})$ and Pf neurons demonstrating cortical projections to cingulate, premotor, and anterior parietal cortices. ${ }^{54,56}$ Groenewegen and Berendse ${ }^{54}$ earlier proposed that the thalamic intralaminar projections provide an anatomical substrate for interactions among relatively segregated cortico-striatopallidal-thalamocortical loop pathways under the control of the prefrontal cortex. In a comprehensive study of intralaminar thalamic connections across the rat forebrain, van der Werf et al. ${ }^{53}$ have shown that the intralaminar nuclei collectively provide comprehensive connections to large cerebral networks roughly corresponding to the cortico-striatopallidalthalamocortical loop systems described in nonhuman primates ${ }^{57}$ including a visceral, motor, limbic and cognitive loop. They propose that the access of prefrontal and brainstem arousal pathways to these neurons provide a mechanism for linking sensory and motor activity to awareness. Consistent with the above proposed mechanism for arousal regulation and selective network activation, Shirvalkar et al..$^{58}$ demonstrated that electrical stimulation of CL increased generalized arousal and recognition memory performance in intact rats. In a series of studies, Mair and colleagues have demonstrated that lesions in the rostral intralaminar region of the rat (centered on CL) produce broad deficits in delayed conditional discrimination across sensory modalities. ${ }^{59}$ In addition, similar lesions of the rat anterior intralaminar nuclei produce deficits in initiating motor behavior. ${ }^{60} \mathrm{~A}$ unifying link across these observations (discussed below) is provided by the recent observation that intracellular recordings in cortex, thalamus, and striatal neurons associate brief increases in multi-unit firing rates in the $30-50 \mathrm{~Hz}$ frequency range typically observed during attentional or memory delays, with shifts of input to individual neurons based on increased rates of bombardment by postsynaptic potentials (balanced excitatory and inhibitory inputs ${ }^{61,62}$ ). 


\section{Role of Sensory Inputs and Pre-motor Attention Signals}

In addition to shifts of firing rates in the central thalamus associated with changes of vigilance level, holding of behavioral sets, such as memory delays, and the broad state changes associated with shifts into wakeful or REM sleep states, electrophysiological recordings also demonstrate that the central thalamic neurons respond to specific sensory and premotor signals. Central thalamic neurons show responses to several brainstem reflex pathways that associate patterned input from the sensory environment, such as acoustic startle, ${ }^{8}$ optokinetic, and vestibular stimuli, ${ }^{63,64}$ noxious tactile stimuli, $^{9}$ and premotor signals including an oculomotor efference copy signal. ${ }^{5,65,66}$ Among the sensory signals detected in the anterior intralaminar nuclei, an alerting response related to the elementary startle response and orienting behavior has been characterized in cats identified as the central lateral elicited wave, $\mathrm{CL}_{\mathrm{E}} \cdot{ }^{8}$ This potential is similar to the $\mathrm{PGO}_{\mathrm{E}}$ wave, a potential modulated by external events thought to reflect environmental monitoring, and both are observed across the sleep-wake cycle. Both $\mathrm{CL}_{\mathrm{E}}$ and $\mathrm{PGO}_{\mathrm{E}}$ resist habituation and persist long after peripheral acoustic startle responses or orienting behaviors terminate. The $\mathrm{CL}_{\mathrm{E}}$ wave, like the $\mathrm{PGO}_{\mathrm{E}}$ wave, likely reflects a widely broadcast signal originating from brainstem circuits evolved to capture organismal behavior. During wakefulness this signal may be used to reset patterns of activation and interrupt ongoing behavioral sets. CLE waves are observed as spontaneous events and may be consistent with eye movements, spontaneous attentional shifts, or other internally organized alerting responses. Signals recorded from the posterior intralaminar regions of the primate $\mathrm{Cm}-\mathrm{Pf}$ complex similarly reflect behaviorally relevant sensory events, onset of sensory cues, ${ }^{44}$ or attentional orienting per se ${ }^{45}$

The evidence of an oculomotor signal within the anterior and posterior intralaminar regions $\mathrm{s}^{5,65}$ is of particular importance because of the close evolution of the frontal lobe executive control systems and primate oculomotor system. ${ }^{5,46,67}$ Because these functional controls have co-evolved in the same brain regions in the human and nonhuman primate brain they share many neurophysiological mechanisms. Modeling studies have proposed that transient signals, such as corollary discharges around eye movements, may initiate the formation or dissolution of maintained elevations of cortical firing rates as discussed above, providing a potential mechanistic link for the appearance of these signals within the same thalamic populations that facilitate increased levels of overall activity in response to task demands. ${ }^{68,69}$ Gutkin et al. ${ }^{68}$ proposed a model of recordings of cortical delay period activity in which onset of increased cortical firing rates (persistent activity) occurred with brief synchronous transients interpreted in the model as efference copy signals of saccades and other motor events. Physiological studies have demonstrated such interactions of persistent activity supporting working memory and repetitive premotor signals, e.g., during memory delay tasks a transient signal related to the eye movements appeared to terminate the delay period activity ${ }^{66}$ Other models also propose that such transient inputs to local cortical networks exhibiting persistent activity may terminate the delay period activity ${ }^{69}$

Thus the alerting, sensory orienting, and corollary discharge signals recorded in the central thalamus may play an important role in turning on and off patterns of broad neuronal activation across the cerebrum during wakefulness. In support of this hypothesis, a study of pair recordings from the central thalamus and regions of extrastriate visual cortex obtained from an awake behaving monkey demonstrate that both transient activation around alerting cues and saccades produce coherent activation across these corticothalamic connections within the $20-$ to $80-\mathrm{Hz}$ frequency range. ${ }^{119}$ Importantly, this finding was specific for neuronal populations that show similar changes in the background thalamic local field potential during short-term focusing of attention. These findings thus provide a link between the arousal regulation of the forebrain by the central thalamus and the anatomical specializations of the central thalamus that allow it to capture attention by using brainstem alerting mechanisms and corticothalamic inputs from the prefrontal cortex. Similar effects are seen with the orienting response in the EEG, which produces a brief increase in power in the approximately $40-\mathrm{Hz}$ frequency range. ${ }^{70}$

\section{Microcircuit and Biophysical Mechanisms Supporting Cortical and Striatal Activation by the Central Thalamus}

Recent studies point to a specific and very general mechanism by which the high frequency impulses emanating from the intralaminar neurons to the cerebral cortex and striatum may support broad network activations during wakefulness. Inputs from the anterior intralaminar nuclei have a unique pattern of innervation of the cortical microcircuit ${ }^{71}$ and specialization of contacts made within the striatum. ${ }^{72}$ Most of the neurons from the anterior intralaminar region synapse in layer I on the apical dendrites of pyramidal cells with cell bodies located in layers II-III and layer V. Jones $^{73}$ has redefined the intralaminar and other thalamic subdivisions into two classes of neurons on the 
basis of differential calcium-binding protein expression. One class, the "core" neurons, express parvalbumin and synapse in the middle, or granular, layers of the cortical microcircuit with area-specific cortical projections. The other class, the "matrix" neurons express calbindin $\mathrm{D}_{28 \mathrm{~K}}$ and project to Layer I across relatively wide cortical territories. In both humans ${ }^{74}$ and nonhuman primates, ${ }^{73}$ intralaminar subdivisions are densely populated with matrix neurons (CL, Pc, Pf, but not $\mathrm{Cm}$, which is densely parvalbumin staining). The matrix neurons are proposed to act collectively as a functional system that organizes global patterns of corticothalamic synchronization. ${ }^{73}$ Neurons within these intralaminar regions may synapse on cell bodies within layers $\mathrm{V}$ and VI as well as Layer $\mathrm{I}^{71}$ and this pattern of dual innervation within the cortical column has been proposed to support a biophysical coincidence detection mechanism that facilitates inputs from the specific thalamic inputs to the granular layers. ${ }^{75,76}$ In studies of the apical dendrites of Layer V and Layer II/III cortical neurons, Larkam et al..$^{77,78}$ have demonstrated that co-activation within a narrow temporal window of the apical dendrites (in Layer I) and soma of Layer $\mathrm{V}$ pyramidal neurons induces burst firing through a back-propagating action potential that originates in the soma and allows for normally subthreshold inputs in the apical dendrites to become spike generating. Similar changes in dendritic electrogenesis of Layer II/III neurons could support this mechanism. ${ }^{78}$ Consistent with these proposals, combined stimulation of CL and the ventrobasal nucleus (a specific thalamic relay nucleus projecting into cortical Layer IV) in mouse corticothalamic slice generates a supralinear summation of local evoked potentials. ${ }^{79}$ Depolarization of the supragranular layer dendrites by intralaminar afferents has been proposed to promote sustained cortical activity ${ }^{80,81}$ NMDA-mediated long-term potentiation, ${ }^{81,46}$ and other mechanisms of synaptic facilitation. ${ }^{82}$ Thus, the anatomical specializations of the matrix neuronrich intralaminar and paralaminar regions of central thalamic thalamus provide a unique substrate for support of distributed cortical persistent activity. Consistent with these in vitro and anatomical studies, recent in vivo electrical stimulation studies show that electrical stimulation of CL generate broad activation of $c-f o s$ across all cortical layers and a unique pattern of upregulation of zif268 (an immediate early gene associated with long-term potentiation) in supragranular and infragranular layers consistent with these mechanisms. ${ }^{58}$

Central thalamic inputs to the striatum are also specialized and recent studies have shown that the central lateral nucleus of the anterior intralaminar group projects to the medium spiny striatal neuron (MSN) contacting the dendritic spine. ${ }^{72}$ These excitatory inputs to the striatal neuron may act as primary drivers along with descending corticostriatal inputs. The MSN is an important link in the control of anterior forebrain dynamics and its activity levels may play a key role in many neurological disorders of consciousness (see discussion below). In the Steriade and Glenn ${ }^{12}$ studies discussed above, inputs to the striatum from CL/Pc neurons had a slower conduction velocity $(3 \mathrm{~m} / \mathrm{s}$ compared with $11 \mathrm{~m} / \mathrm{s}$ for cortically projecting neurons) suggesting the possibility that the timing of inputs from these regions may be controlled to facilitate selective activation as suggested by earlier proposals. ${ }^{54}$

Experimental studies support the view that activation of both the cortical and striatal neurons by inputs arising from the central thalamus may facilitate the generation of a phenomenon identified in in vitro and anesthetized animal preparations as the "UP state". During the UP state, membrane potential show relatively depolarized baselines and fast firing of the neuron. The UP state is a network-dependent phenomena that reflects broad increases in excitatory and inhibitory postsynaptic potentials bombarding the dendritic arbor of neurons across large cerebral networks linking cortex, thalamus, and basal ganglia. ${ }^{61,62,83}$ From a functional point of view, the inputs to central thalamus and patterns of activation of central thalamic neurons during attentive behaviors suggest that they act as gain controls for distributed processes throughout the cortex, thalamus, and basal ganglia. The common mechanism may be shifts in activation across the networks increasing an overall output of balanced excitatory and inhibitory synaptic barrage associated with an UP state-like phenomena during wakefulness. ${ }^{61,83}$ Firing rates of multi-unit activity during UP states recorded in vitro and under anesthesia averages $35-50 \mathrm{~Hz}$, and the activity pattern is proposed to underlie the typical appearance of similar firing rates in behaving animals carrying out cognitive tasks as discussed above. ${ }^{61,62}$ A related common signature of these phenomena may be increases in local field potentials in the band $20-80 \mathrm{~Hz}$ that could reflect increased synaptic drive of cortical inhibitory interneuron populations ${ }^{84}$ and synchronization of signals in the cortico-cortical, ${ }^{46}$ corticothalamic, ${ }^{48,75,76}$ corticostriatal, ${ }^{85}$ and thalamostriatal pathways. ${ }^{85}$ Importantly, a recent experimental study has demonstrated that thalamocortical projections are significantly more efficient in producing cortical UP states than cortico-cortical excitatory connections; ${ }^{86}$ the central thalamic projections to both cortex and striatum may have similar properties, although this is not known. 
The unique connectivity of central thalamic neurons and their physiological specializations suggest that they may contribute to broad formation of UP statelike transition of firing rates seen across cerebral networks in vivo during organized behaviors. Facilitating the transition to UP states and their maintenance may be the fundamental mechanism of arousal regulation through the central thalamus that allows for: 1) adjustment of gain across multiple pathways of sensorimotor integration associated with varying levels of vigilance, interest, motivation, and other state factors and 2) rapid capture and redirection of behavior through powerful bias controls facilitated through activation of the central thalamus by the frontal executive systems using premotor signals that direct attention to intended movements through a broad corollary discharge mechanism. Similarly, biasing of attention and goal-directed action may be achieved by triggering UP states across cerebral networks through activation of dedicated reflex pathways from the sensory periphery that access the central thalamus. Using this picture as a working model, it is possible to organize many disparate observations associated with neurological disorders of consciousness and their phenomenology as reviewed below.

\section{Contributions of the Central Thalamus to Neurological Disorders of Consciousness}

It is well established that the central thalamus plays an important role in neurological disorders of consciousness. ${ }^{87}$ The brief review below links this role of the central thalamus to pathophysiological mechanisms that produce both persistent and paroxysmal impairments of arousal regulation after severe brain injury. An interpretative framework is then proposed to explain underlying mechanisms for interventions that improve neurological function in patients with disorders of consciousness.

\section{Focal Injuries to the Central Thalamus}

Direct injuries to the central thalamus can alone produce global disturbances of consciousness. ${ }^{88-90}$ If unilateral, these lesions may produce hemispatial unawareness ${ }^{91}$ or altered states of consciousness similar to acute mania or delirium. ${ }^{92}$ Restricted bilateral injuries to the central thalamus may produce acute coma indicating the important role these structures play in a normal alert wakeful state. Recovery of cyclical arousal patterns with periods of eye opening occurs rapidly with these lesions and is usually present within $48 \mathrm{~h}$, again emphasizing that these neurons are not the primary sources of ascending control of arousal state per se. However, unlike brainstem lesions that produce coma following focal injuries, ${ }^{93}$ the recovery of consciousness in terms of goal-directed behavior and communication skills is very slow following bilateral central thalamic injuries ${ }^{89,90}$ and uncertain. ${ }^{88}$ These clinical observations are consistent with experimental studies that demonstrate that neither the central thalamus nor the basal forebrain are indispensable for maintaining general arousal associated with some recovery of EEG cycling and wakefulness, ${ }^{14,94}$ suggesting that parallel basal forebrain and thalamic mechanisms and direct brainstem to cortex pathways are pleuripotent for maintaining cerebral arousal (see Ref. 23 for review).

The large and enduring behavioral effects of small, central, thalamic injuries can be understood in the context of studies that demonstrate that focal ischemic injuries within the central thalamus can produce marked reduction in cerebral metabolism across the entire ipsilateral hemisphere as measured using fluorodeoxglucose positron emission tomography. ${ }^{95,96}$ This effect reflects a crossed (trans-) synaptic downregulation of distant neuronal populations connected to the central thalamus (known also as diaschisis ${ }^{97}$ ). The crossed synaptic downregulation results from the loss of excitatory inputs to remote brain regions, and these changes in cerebral blood flow only marginally indicate marked reductions in neuronal firing rates in the distal structures. ${ }^{98}$ The loss of excitatory drive to neuronal populations in such instances produces a passive form of inhibition (disfacilitation) hyperpolarizing neuronal membrane potentials as a result of withdrawal of excitatory synaptic inputs that then allow remaining leak currents (principally potassium) to dominate. ${ }^{99}$

The profound effects of central thalamic lesions can be understood simply as a consequence of their unique geometry; the neurons have many point to point connections across the corticothalamic system and through the striatal loop connections of the anterior forebrain (as reviewed above). A quantitative result underscoring this point is the finding, in a complete multidimensional scaling model of the entire cat thalamocortical system ${ }^{100}$ that places the anterior intralaminar nuclei (CL, Pc, and central medial) in one cluster together, centered among all other segregated thalamocortical loop systems (frontal, limbic, temporal, and parietal). This positioning can be interpreted as a consequence of their relatively shortest path and multiple connections across each of the other clusters (with a tendency to be closer to the frontal/limbic region of the cortex, which is more strongly connected with the striatum). In this context, the broad effects 
of withdrawal of central thalamic excitation can help to explain the surprisingly long-lasting impairments of human frontal lobe executive functions correlated with discrete injury to $\mathrm{CL}^{101}$ and other thalamic injuries specifically involving the rostral intralaminar region. ${ }^{88,102}$

In addition to persistent decreases in neuronal firing rates across the cerebrum, discrete injuries to the central thalamus may also lead to global functional impairment through different forms of abnormal hypersynchronous activity. ${ }^{47,87}$ Focal injuries within the central thalamus are also associated with production of epileptic seizures, typically variations of absence seizures ${ }^{90}$ and other paroxysmal phenomena sharing features similar to catatonia, dystonia, and parkinsonism, and responsive to pharmacologic agents used to treat those disorders (reviewed in Refs. 47,103).

\section{Contribution of Central Thalamus to Disorders of Consciousness Following Multifocal Diffuse Brain Injuries}

The far more frequent cause of neurological disorders of consciousness following severe brain injury is widespread deafferentation of the central thalamus because of widespread neuronal death or disconnection. Consistent with the discussion above, this too is the simple consequence of the unique geometry of central thalamic connections but is not well appreciated in the context of pathologies underlying global disorders of consciousness, ${ }^{103}$ such as the permanent vegetative state ${ }^{104}$ and enduring the minimally conscious state (MCS). ${ }^{105}$

Three very common mechanisms of injury that may bilaterally involve the central thalamus arise in the setting of multifocal head trauma, large hemispheric strokes, and hemorrhages: 1) diffuse axonal injury, 2) downward displacement of thalamus and brainstem as a result of brain swelling (known as "herniation"), with or without 3) en passant focal ischemic injuries to the brainstem, midbrain, thalamus, or basal ganglia. Diffuse axonal injury results in widespread disconnection across the cerebrum with a final common finding of secondary neuronal death of thalamic neurons indexing outcomes. Even in the most severe multifocal brain injuries associated with permanent VS, neuronal death in the thalamus is the most common pathological finding. In a study ${ }^{104}$ of nontraumatic injuries associated with permanent VS (mostly anoxia following cardiac arrest), all patients showed severe bilateral thalamic damage, which was not invariably associated with diffuse cortical damage (64\% of cases). In pathological studies of brains from patients who suffered traumatic injuries and remained in VS for at least 3 months prior to death, a similar finding of widespread thalamic neuronal loss was found with less neocortical involvement (only 11\% with diffuse neocortical cell loss). In a recent follow-up study, the detailed analysis of thalamic nuclei revealed that when autopsy studies were compared with controls, damage to central thalamic structures indexed behavioral outcomes from moderate disability to VS with initial involvement of the anterior intralaminar structure and progressive cell loss ventrally and posteriorly with worsening outcome. ${ }^{106}$ These findings reflect the broad effects of neuronal death and disconnection with a rostrocaudal gradient consistent with the more diffuse and widespread point to point connections of the anterior intralaminar/paralaminar regions. Herniation injuries with or without evidence of focal ischemic lesions within the central thalamus (or closely linked, subcortical, neuronal populations) can produce profound functional downregulation across the entire ipsilateral cerebral hemisphere and possibly bilateral metabolic dysregulation. ${ }^{95,96,107}$ Similarly, persistent global cerebral dysfunction in patients may be associated with abnormal capture by pathological rhythmic activity in the central thalamus produced in frontal cortical or other areas. ${ }^{107}$

\section{Modulation of Central Thalamus and Effects on Disorders of Consciousness}

Disorders of consciousness have generally been considered untreatable if enduring for months or longer. ${ }^{108}$ Recent observations of late improvement in MCS patients with long-standing brain injuries, whether spontaneous, ${ }^{109}$ in response to pharmacologic agents, ${ }^{110}$ or following electrical stimulation, ${ }^{111}$ suggest that circuit mechanisms may be available for improving functional outcomes in some cases.

Taking into consideration findings that some very severely brain-injured patients, who nonetheless show some responses to their environment, may have widely preserved cerebral network activation, ${ }^{112}$ Schiff and colleagues sought to determine whether electrical stimulation could restore arousal regulation and promote greater behavioral responsiveness in MCS patients. ${ }^{111-113}$ They proposed that central thalamic deep-brain stimulation (DBS) might substitute for topdown monitoring and regulation of arousal level provided in the intact brain by mesial frontal cortical regions and brainstem inputs (as reviewed above). Application of central thalamic DBS in a 38-year-old man who remained in MCS for 6 years led to restorations of spoken language, oral feeding, and motor control of the upper limbs. These findings suggest that electrical stimulation may approximate the normal role of cortical and subcortical inputs to the central 

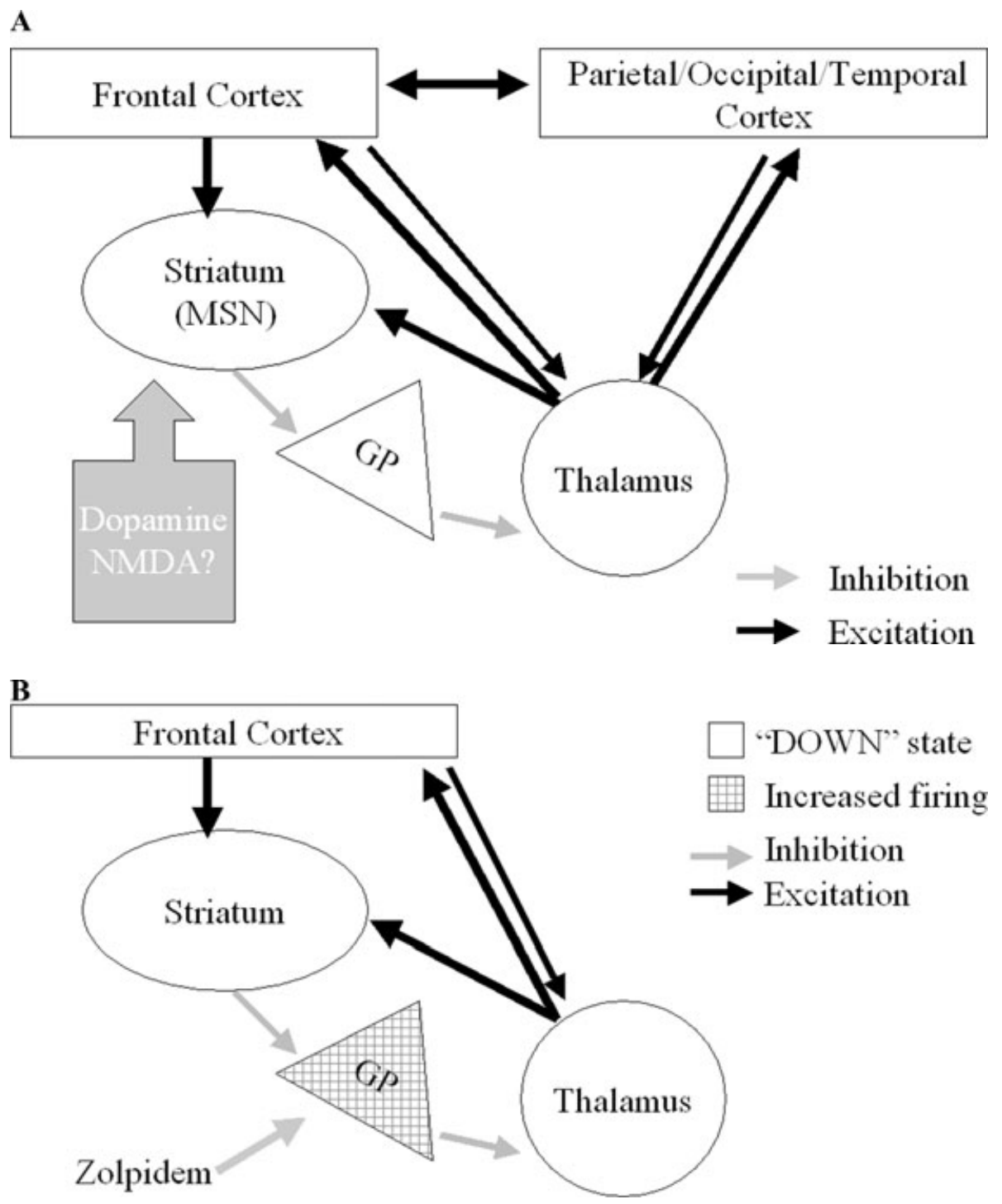

FIGURE 2. (A) Potential circuit mechanisms underlying overlapping responses to dopaminergic agents, NMDA antagonists, sedatives (Ambien, Lorazepam), and central thalamic deep-brain stimulation (DBS) in severe brain injuries. A variety of pharmacologic agents have been shown to improve responsiveness in patients with global disorders of consciousness. Connections across the anterior forebrain may play a key role in the response to multiple pharmacologic agents through modulation of a common circuit abnormality arising in the setting of multifocal neuronal cell death. Thalamocortical and thalamostriatal output from the central thalamus may be reduced as a result of die back of connections across multiple cortical regions from neuronal death or axonal disruption. These thalamic afferents appear to have a preferential role in activating broad networks across the cortex and producing activation of striatal neurons (see text). (B) Proposal for circuit mechanism underlying paradoxical Ambien (zolpidem) response. ${ }^{116}$

thalamus that act to adjust neuronal firing rates in these neurons to regulate cognitive effort and maintain brain metabolic activity in normal wakeful states. ${ }^{47,58,113} \mathrm{In}$ the severely injured brain, DBS of the central thalamus may counteract strong passive inhibition of the central thalamus as a result of the widespread loss of incoming axonal afferents from many cerebral structures (thus effectively overcoming the major problem of loss of excitatory contacts across the dendritic arbor of these neurons). The observation noted above 
that anterior intralaminar regions show progressive neuronal loss in correlation with severity of cognitive impairment following brain injury supports this argument. ${ }^{106}$ Moreover, functional studies of changes in resting metabolism after diffuse axonal injury show a specific pattern consistent with impaired function of the anterior forebrain, including marked downregulation of metabolic rates in mesial frontal structures and the thalamus bilaterally. ${ }^{114}$

The results of the central thalamic DBS studies can be considered alongside other interventions that in some patients facilitate behavioral responsiveness after severe brain injury leading to MCS or severe disability. ${ }^{110,115,116}$ Both classical dopaminergic agents (L-Dopa, Bromocriptine) and the mixed dopamine agonist/NMDA-channel antagonist Amantidine have been reported to facilitate recovery of function. ${ }^{15}$ Taken together with the DBS results described above, these observations are consistent with a model that identifies dysfunction across a broad anterior forebrain system primarily arising from widespread deafferentation of cortical neurons producing reduced levels of global neuronal activity across the brain and causing relatively increased deafferentation of the central thalamus (resulting from the geometry of their point to point connections). As illustrated in FigURE 2A, an important point of vulnerability within the anterior forebrain is the medium spiny neuron (MSN) of the striatum. These neurons have a high threshold UP state and thus do not fire easily, requiring both a sufficient level of dopaminergic input and background synaptic activity from corticostriatal and thalamostriatal inputs. ${ }^{117}$ MSN output is necessary to oppose tonic activity of the globus pallidus interna (GPi), which acts as a strong inhibitory influence on the thalamus. Neurons in the central lateral nucleus of the anterior intralaminar regions that undergo significant neuronal loss following multifocal brain injuries also provide input to the dendritic spines of the MSNs and may specifically contribute to reducing their output following injury. Thus, both dopaminergic agents and direct activation of the central thalamus may act to reverse downregulation across the anterior forebrain systems produced by severe brain injury. Dopaminergic agents strongly modulate both striatal and mesial frontal systems, whereas central thalamic neurons provide direct excitatory (glutamatergic) activation of the supragranular cortical layers and MSNs. Importantly, as noted above, thalamic neurons have a preferential role for generating cortical UP states. ${ }^{86}$ Reduced pallidal inhibition with increased MSN output may allow the promotion of cortical UP states via release of strong thalamocortical activation.
This model of altered network function that emphasizes impaired function of anterior forebrain loop systems following severe brain injury makes another specific prediction. ${ }^{116}$ As shown in Figure 2B, this model can offer an explanation of the very paradoxical observation that some severely brain-injured patients are "awakened" and show marked behavioral facilitation with the sedative agent zolpidem. ${ }^{10}$ Zolpidem binds selectively to the alpha- 1 subunit of the GABA-A receptor, which is highly expressed in the GPi, and microinfusion of zolpidem into GPi can produce behavioral changes consistent with inactivation of the structure. ${ }^{18}$ Most of the patients who have demonstrated these effects have suffered hypoxic injuries, which produce significant impairment of MSNs. Zolpidem may act to suppress disinhibited GPi neurons allowing reactivation of thalamocortical and thalamostriatal afferents re-establishing function in the anterior forebrain network. ${ }^{116}$ Reversal of marked hypometabolism in the frontal lobes and thalami in association with behavioral improvements with zolpidem supports this inference. ${ }^{110}$ Similar effects with lorazepam, a less-selective agent, may arise via the same mechanism (unpublished observations).

\section{Conclusions}

As reviewed above, the neurons in the central thalamus are uniquely positioned to control dynamic patterns of activation across cerebral networks. These neurons may drive both cortical and striatal neurons to transition into the equivalent of an UP state during wakeful behaviors, organizing very long-range connections in the forebrain. Transient activation of the same thalamic neurons appears in response to premotor corollary discharge signals and bottom-up sensorimotor reflexes originating in the brainstem, which likely briefly facilitate broad synchronization through a similar mechanism. Damage to this efficient system of forebrain activation leads to immediate and global impairments of consciousness and goal-directed behavior. Understanding the contributions of the central thalamus to arousal regulation may aid our rethinking and reformulating of how to approach the problems posed by disorders of consciousness. Based on their unique anatomical and physiological properties, neurons within the central thalamus are suggested as targets of interventions aimed at restoring function at the circuit level for patients with disorders of consciousness. The observations of individual patients' responses to DBS, sedative, and other drugs reviewed above emphasize the key role of the central thalamus in circuit disturbances affecting large-scale forebrain dynamics. 


\section{Competing Interests Statement}

One of the references cited in the text, Ref. 111, describes work that was, in part, funded by a company, IntElect Medical, Inc., in which Cornell University has part ownership; through the licensing of technology, the author is a listed Cornell inventor and may benefit in the future from commercialization of intellectual property owned by Cornell.

\section{Conflicts of Interest}

The author declares no conflicts of interest.

\section{References}

1. Steriade, M., E. Jones \& D. McCormick. 1997. Thalamus. Elsevier Publishers.

2. Parvizi, J. \& A. Damasio. 2001. Consciousness and the brainstem. Cognition 79: 135-160.

3. Norman, D.A. \& T. Shallice. 1986. Attention to action: Willed and automatic control of behaviour. In Consciousness and self-regulation, Vol. 4. R.J. Davidson, G.E. Schwartz \& D. Shapiro, Eds.: 1-18. Plenum Press. New York.

4. Rizzolatti, G. et al. 1987. Reorienting attention across the horizontal and vertical meridians: evidence in favor of a premotor theory of attention. Neuropsychologia 25: 31-40.

5. Schlag-Rey, M. \&J. Schlag. 1984. Visuomotor functions of central thalamus in monkey. I. Unit activity related to spontaneous eye movements. J. Neurophysiology 40: 1149-1174.

6. Posner, M.I. 1978. Chronometric explorations of mind. Erlbaum Press.

7. Parasuraman, R., J.S. Warm \& J.E. See. 1999. Brain systems of vigilance. In The Attentive Brain. Parasuraman Ed.: MIT Press.

8. SANFORD, L.D. et al. 1992. Varying expressions of alerting mechanisms in wakefulness and across sleep states. Electroencephalogr. Clin. Neurophysiol. 82: 458-468.

9. Krout, K.E., R.E. Belzer \& A.D. Loewy. 2002. Brainstem projections to midline and intralaminar thalamic nuclei of the rat. J. Comp. Neurol. 17: 53-101.

10. Morison, R.S. \& E.W. Dempsey. 1942. A study of thalamocortical relations. Am. J. Physiol. 135: 281-292.

11. Moruzzi, G. \& H.W. Magoun. 1949. Brainstem reticular formation and activation of the EEG. Electroencephalogr. Clin. Neurophysiol. 1: 455-473.

12. Steriade, M. \& L.L. Glenn. 1982. Neocortical and caudate projections of intralaminar thalamic neurons and their synaptic excitation from midbrain reticular core. J. Neurophysiol. 48: 352-371.

13. Glenn, L.L. \& M. Steriade. 1982. Discharge rate and excitability of cortically projecting intralaminar neurons during waking and sleep states. J. Neuroscience. 2: 13871404.
14. Steriade, M., R. Gurro Dossi \& D. Contreras. 1993. Electrophysiological properties of intralaminar thalamocortical cells discharging rhythmic (approximately 40 HZ) spike-bursts at approximately $1000 \mathrm{HZ}$ during waking and rapid eye movement sleep. Neuroscience 56: $1-9$.

15. Ribary, U. et al. 1991. Magnetic field tomography of coherent thalamocortical $40-\mathrm{Hz}$ oscillations in humans. Proc. Natl. Acad. Sci. USA 88: 11037-11041.

16. Llinas, R. \& U. Ribary. 1993. Coherent 40-Hz oscillation characterizes dream state in humans. Proc. Natl. Acad. Sci. USA 90: 2078-2081.

17. Gottesmann, G. 1999. Neurophysiological support of consciousness during waking and sleep. Prog. Neurobiol. 59: 469-508.

18. Steriade, M. et al. 1990. Neuronal activities in brainstem cholinergic nuclei related to tonic activation processes in thalamocortical systems. J. Neurosci. 10: 2541-2559.

19. Saper, G.B., T.E. Scammell \& J. Lu. 2005. Hypothalamic regulation of sleep and circadian rhythms. Nature 437: 1257-1263.

20. Smiley, J.F., M. Subramanian \& M.M. Mesulam. 1999. Monaminergic-cholinergi interactions in the primate basal forebrain. Neuroscience 93: 817-829.

21. Sutcliffe, J.G. \& L. DE LeceA. 2002. The hypocretins: setting the arousal threshold. Nat. Rev. Neurosci. 3: 339 349.

22. McCormick, D.A. \& T. BAL. 1997. Sleep and arousal: thalamocortical mechanisms. Annu. Rev. Neurosci. 20: 185-215.

23. PfafF, D. 2005. Brain arousal and information processing. Harvard University Press.

24. Kolmac, C. \& J. Mitrofanis. 1999. Organization of the basal forebrain projection to the thalamus in rats $\mathrm{Neu}-$ rosci. Lett. 272(3): 151-154.

25. Lavoie, B. \& A. Parent. Serotoninergic innervation of the thalamus in the primate: an immunohistochemical study. J. Comp. Neurol. 312: 1-18, 26.

26. Oke, A.F. et al. 1997. Three-dimensional mapping of norepinephrine and serotonin in human thalamus. Brain Res. 763: 69-78.

27. Erro, E., J.L. Lanciego \& J.M. Giménez-Amaya. 1999. Relationships between thalamostriatal neurons and pedunculopontine projections to the thalamus: a neuroanatomical tract-tracing study in the rat. Exp. Brain Res. 127: 162-170.

28. Kinomura, S. et al. 1996. Activation by attention of the human reticular formation and thalamic intralaminar nuclei. Science 271: 512-515.

29. NAito, E. et al. 2000. Fast reaction to different sensory modalities activates common fields in the motor areas, but the anterior cingulate cortex is involved in the speed of reaction. J. Neurophysiol. 83: 1701-1709.

30. NAGAI, Y. et al. 2004. Brain activity relating to the contingent negative variation: an $\mathrm{fMRI}$ investigation. Neuroimage 21: 1232-1241.

31. PAus, T. et al. 1997. Time-related changes in Neural systems underlying attention and arousal during the performance of an auditory vigilance task. Jour. Cognitive Neurosci. 9: $392-408$. 
32. PAus, T. 2000. Functional anatomy of arousal and attention systems in the human brain. Prog. Brain Res. 126: 6577.

33. Vogt, B.A., D.L. Rosene \& D.N. Pandya. 1979. Thalamic and cortical afferents differentiate anterior from posterior cingulate cortex in the monkey. Science 204: 205 207.

34. Barbas, H. \& D.N. Pandya. 1989. Architecture and intrinsic connections of the prefrontal cortex in the rhesus monkey. J. Comp. Neurol. 286: 353-375.

35. Duncan, J. \& A.M. Owen. 2000. Common regions of the human frontal lobe recruited by diverse cognitive demands. Trends Neurosci. 23: 475-483.

36. Paus, T. et al. 1998. Regional differences in the effects of task difficulty and motor output on blood flow response in the human anterior cingulate cortex: a review of 107 PET activation studies. Neuroreport 9: R3747.

37. Rushworth, M.F. et al. 2003. The effect of cingulate cortex lesions on task switching and working memory. J. Cogn. Neurosci 15: 338-353.

38. MOREL, A. et al. 2005. Divergence and convergence of thalamocortical projections to premotor and supplementary motor cortex: a multiple tracing study in the macaque monkey. Eur. J. Neurosci. 21: 1007-1029.

39. Schiff, N.D., S.F. Kalik \& K.P. Purpura. 2001. Sustained activity in the central thalamus and extrastriate areas during attentive visuomotor behavior: correlation of single unit activity and local field potentials. Society for Neuroscience 30st Annual Meeting.

40. Schiff, N.D., A.E. Hudson \& K.P. Purpura. 2002. Modeling wakeful unresponsiveness: characterization and microstimulation of the central thalamus. Society for Neuroscience 31st Annual Meeting Abstract 62.12.

41. Fries, P. et al. 2001. Modulation of oscillatory neuronal synchronization by selective visual attention. Science 291: 1560-1563.

42. Murthy, V.N. \& E.E. Fetz. 1996. Synchronization of neurons during local field potential oscillations in sensorimotor cortex of awake monkeys. J. Neurophysiol. 76: 3968-3982.

43. Pesaran, B., et al. 2002. Temporal structure in neuronal activity during working memory in macaque parietal cortex. Nat. Neurosci. 5: 805-811.

44. Matsumoto, N. et al. 2001. Neurons in the thalamic CM-Pf complex supply striatal neurons with information about behaviorally significant sensory events. J. Neurophysiol. 85: 960-976.

45. Minamimoto, T. \& Kimura, M. 2002. Participation of the thalamic CM-Pf complex in attentional orienting. J. Neurophysiol. 87: 3090-3101.

46. PurpurA, K.P. \& N.D. Schiff. 1997. The thalamic intralaminar nuclei: role in visual awareness. Neuroscientist 3: 8-14.

47. Schiff, N.D. \& K.P. PURPuRA. 2002. Towards a neurophysiological basis for cognitive neuromodulation through deep brain stimulation. Thalamus Related Syst. 2: 55-69.

48. Steriade, M. et al. 1996. Synchronization of fast (30-40 $\mathrm{Hz})$ spontaneous oscillations in intrathalamic and thalamocortical networks. J. Neurosci. 16: 2788-2808.
49. Steriade, M. et al. 1991. Fast oscillations $(20-40 \mathrm{~Hz})$ in thalamocortical systems and their potentiation by mesopontine cholinergic nuclei in the cat. Proc. Natl. Acad. Sci. 88: $4396-4400$.

50. Gimenez-Amaya, J.M. \& E. Scarnati. 1999. The thalamus as a place for interaction between the input and the output of the basal ganglia: a commentary. Jour. Chem. Neuroanat 16: 149-152.

51. Jones, E.J. 2007. The thalamus. 2nd edn. Cambridge University Press.

52. Macchi, G. \& M. Bentivoglio. 1985. The thalamic intralaminar nuclei and the cerebral cortex. In Cerebral Cortex, Vol. 5. E.G. Jones \& A. Peters, Eds.: 355-389. Plenum Press. New York.

53. VAN der Werf, Y.D., M.P. Witter \& H.J. GroeneweGEN. 2002. The intralaminar and midline nuclei of the thalamus. Anatomical and functional evidence for participation in processes of arousal and awareness. Brain Res. Brain Res. Rev. 39: 107-140.

54. Gronewegen, H. \& H. Berendse. 1994. The specificity of the 'nonspecific' midline and intralaminar thalamic nuclei. Trends Neurosci. 17: 52-66.

55. Sidibe, M., J.F. PARE \& Y. SMITH. 2002. Nigral and pallidal inputs to functionally segregated thalamostriatal neurons in the centromedian/parafascicular intralaminar nuclear complex in monkey. J. Comp. Neurol. 447: 286-299.

56. SADikOT, A.F. et al. 1992. Efferent connections of the centromedian and parafascicular thalamic nuclei in the squirrel monkey: a light and electron microscopic study of the thalamostriatal projection in relation to striatal heterogeneity. J. Comp. Neurol. 320: 228-242.

57. Alexander, G.E., M.R. DeLong \& P.L. Strick. 1986. Parallel organization of functionally segregated circuits linking basal ganglia and cortex. Ann. Rev. Neurosci. 9: 357-381.

58. Shirvalkar, P. et al. 2006. Cognitive enhancement with central thalamic electrical stimulation. Proceedings of the National Academy of Sciences 103: 1700717012.

59. BuRK, J.A. \& R.G. MAIR. 1998. Thalamic amnesia reconsidered: excitotoxic lesions of the intralaminar nuclei, but not the mediodorsal nucleus, disrupt place delayed matching-to-sample performance in rats (Rattus norvegicus). Behav. Neurosci. 112: 54.

60. BuRK, J.A. \& R.G. MAIR. 2001. Effects of intralaminar thalamic lesions on sensory attention and motor intention in the rat: a comparison with lesions involving frontal cortex and hippocampus. Behav. Brain Res. 123: 49-63.

61. McCormick, D.A. et al. 2003. Persistent cortical activity: mechanisms of generation and effects on neuronal excitability. Cereb Cortex. 13: 1219-1231.

62. Shu, Y., A. Hasenstaub \& D.A. McCormick. 2003. Turning on and off recurrent balanced cortical activity. Nature 423: 288-293.

63. ZHU,J.J. \& F.S. Lo. 1998. Control of recurrent inhibition of the lateral posterior-pulvinar complex by afferents from the deep layers of the superior colliculus of the rabbit. J. Neurophysiol. 80: 1122-1131.

64. Shiroyama, T. et al. 1999. Projections of the vestibular nuclei to the thalamus in the rat: a Phaseolus vulgaris leucoagglutinin study. J. Comp. Neurol. 407: 318-332. 
65. Schlag, J. \& M. Schlag-Rey. 1971. Induction of oculomotor responses from thalamic internal medullary lamina in the cat. Exp. Neurol. 33: 498-508.

66. Wyder, M.T., D.P. Massoglia \& T.R. Stanford. 2003. Quantitative assessment of the timing and tuning of visual-related, saccade-related, and delay period activity in primate central thalamus. J. Neurophysiol. 90: 20292052.

67. Corbetta, M. \& G.L. Shulman. 2002. Control of goaldirected and stimulus-driven attention in the brain. Nat. Rev. Neurosci. 3: 201-215.

68. GutKin, B.S. et al. 2001. Turning on and off with excitation: the role of spike-timing asynchrony and synchrony in sustained neural activity. Comput. Neurosci 11: 121134.

69. Brunel, N. \& X.J. WANG. 2001. Effects of neuromodulation in a cortical network model of object working memory dominated by recurrent inhibition. J. Comput. Neurosci. 11: 63-85.

70. Kisley, M.A. \& Z.M. CORNwELL. 2006. Gamma and beta neural activity evoked during a sensory gating paradigm: effects of auditory, somatosensory and cross-modal stimulation. Clin. Neurophysiol. 117: 2549-2563.

71. Macchi, G. 1993. The intralaminar system revisited. In Thalamic Networks for Relay and Modulation. D. Miniacchi et al. Eds.: 209-228. Pergamon. Oxford.

72. Lacey, G.J., J.P. Bolam \& P.J. Magill. 2007. Novel and distinct operational principles of intralaminar thalamic neurons and their striatal projections. J. Neurosci. 27: 43744384.

73. Jones, E.G. 2001. The thalamic matrix and thalamocortical synchrony. Trends Neurosci 24: 595-601.

74. Munkle, M.C., H.J. Waldvogel \& R.L. Faull. 2000. The distribution of calbindin, calretinin and parvalbumin immunoreactivity in the human thalamus. J. Chem. Neuroanat. 19: 155-173.

75. Llinas, R. et al. 1994. Content and context in temporal thalamocortical binding. In Temporal Coding in the Brain. G. Buzsaki et al. Eds.: 252-272. Springer-Verlag. Heidelberg.

76. LLinÁs, R. et al. 1998. The neuronal basis for consciousness. Phil. Trans. R. Soc. London 153: 1841-1849.

77. Larkum, M.E., J.J. Zhu \& B. Sakmann. 1999. A new cellular mechanism for coupling inputs arriving at different cortical layers. Nature 398: $338-341$.

78. LaRkum, M.E. et al. 2007. Dendritic spikes in apical dendrites of neocortical layer 2/3 pyramidal neurons. J. Neurosci. 27: 8999-9008.

79. Llinas, R.R., E. LeZnik \& F.J. Urbano. 2002. Temporal binding via cortical coincidence detection of specific and nonspecific thalamocortical inputs: a voltage-dependent dye-imaging study in mouse brain slices. Proc. Natl. Acad. Sci. 99: 449-454.

80. Vogt, B.A. 1991. The role of layer 1 in cortical function. In Cerebral Cortex, Vol. 9. E.G. Jones \& A. Peters, Eds.: 49-80. Plenum Press. New York.

81. MAIR, R. 1994. On the role of thalamic pathology in diencephalic amnesia. Rev. Neurosci. 5: 105-140.

82. MaCormick, D.A. \& M. von Krosigk. 1992. Corticothalamic activation modulates thalamic firing through glutamate "metabotropic" receptors. Proc. Natl. Acad. Sci. 89: $2774-2778$.

83. Haider, B. et al. 2007. Enhancement of visual responsiveness by spontaneous local network activity in vivo. J. Neurophysiol. 97: 4186-4202.

84. Traub, R.D., J.G.R. Jefferys \& M.A. Whittington. 1999. Fast Oscillations in Cortical Circuits. MIT Press.

85. Blackwell, K.T., U. Czubayko \& D. Plenz. 2003. Quantitative estimate of synaptic inputs to striatal neurons during up and down states in vitro. J. Neurosci. 23: 91239132.

86. Rigas, P. \& M.A. Castro-Alamancos. 2007. Thalamocortical up states: differential effects of intrinsic and extrinsic cortical inputs on persistent activity. J. Neurosci. 27: 4261-4272.

87. Schiff, N.D. \& F. Plum. 2000. The role of arousal and 'gating' systems in the neurology of impaired consciousness. Journal of Clin. Neurophysiol. 17: 438-452.

88. Castaigne, P. et al. 1981. Paramedian thalamic and midbrain infarcts: clinical and neuropathological study. Ann. Neurol. 10: 127-148.

89. KrolaK-Salmon, P. et al. 2000. Total recovery after bilateral paramedian thalamic infarct. Eur. Neurol. 44: 216-218.

90. von Domburg, P.H., H.J. Ten DonkelaAr \& S.L. NoterMANS. 1996. Akinetic mutism with bithalamic infarction. Neurophysiological correlates. J. Neurol. Sci. 139: 5865.

91. Heilman, K.M. et al. 1987. Attention: behavioral and neural mechanisms. In Handbook of Physiology-The Nervous System V. 461-481.

92. Bogousslavsky, J. et al. 1988. Manic delirium and frontallike syndrome with paramedian infarction of the right thalamus. J. Neurol. Neurosurg Psychiatry 51: 116119.

93. Parvizi, J. \& A.R. Damasio. 2003. Neuroanatomical correlates of brainstem coma. Brain 126: 1524-1536.

94. Dringenberg, H.C. \& M.C. Olmstead. Integrated contributions of basal forebrain and thalamus to neocortical activation elicited by pedunculopontine tegmental stimulation in urethane-anesthetized rats. Neuroscience 119: 839-853.

95. Szelies, B. et al. 1991. Widespread functional effects of discrete thalamic infarction. Arch. Neurol. 48: 178182.

96. Gaselli, R.J., N.R. Graff-Radford \& K. Rezai. 1991. Thalamocortical diaschisis: single-photon emission tomographic study of cortical blood flow changes after focal thalamic infarction. Neuropsych. Neuropsych. Behav. Neurol. 4: 193-214.

97. Nguyen, D.K. \& M.I. Botez. 1998. Diaschisis and neurobehavior. Can. J. Neurol. Sci. 25: 5-12.

98. Gold, L. \& M. LAURitzen. 2002. Neuronal deactivation explains decreased cerebellar blood flow in response to focal cerebral ischemia or suppressed neocortical function. Proc. Natl. Acad. Sci. 99: 7699-7704.

99. Timofeev, I., F. Grenier \& M. Steriade. 2001. Disfacilitation and active inhibition in the neocortex during the natural sleep-wake cycle: an intracellular study. Proc. Natl. Acad. Sci. 98: 1924-1929. 
100. ScannelL, J.W. et al. 1999. The connectional organization of the cortico-thalamic system of the cat. Cereb Cortex 9: 277-299.

101. VAN Der WeRF, Y.D. et al. 1999. Neuropsychological correlates of a right unilateral lacunar thalamic infarction. J. Neurol. Neurosurg. Psychiatry 66: 36-42.

102. VAN DER WERF, Y.D. et al. 2003. Deficits of memory, executive functioning and attention following infarction in the thalamus; a study of 22 cases with localised lesions. Neuropsychologia 41: 1330.

103. SCHIFF, N.D. 2005. Modeling the minimally conscious state: measurements of brain function and therapeutic possibilities. Progress Brain Res. 150: 477-497.

104. Adams, J.H., D.I. Graham \& B. JennetT. 2000. The neuropathology of the vegetative state after acute insult. Brain 123: 1327-1338.

105. JennetT, B. et al. 2001. Neuropathology in vegetative and severely disabled patients after head injury. Neurology 56: $486-490$.

106. MAXWELL, W.L., et al. 2006. Thalamic nuclei after human blunt head injury. J. Neuropathol. Exp. Neurol. 65: 478488.

107. Kobylarz, E.J. et al. 2005. Power spectrum and coherence analysis of the electroencephalogram from two minimally conscious patients with severe asymmetric brain damage. Society for Neuroscience $35^{\text {th }}$ Annual Meeting, Abstract.

108. Lombardi, F. et al. 2002. The Cochrane Database of Systematic reviews. Issue 2, Art. No.; D001427. DOI:10.1002/14651858.CD001427.

109. Voss, H.U. et al. 2006. Possible axonal regrowth in late recovery from minimally conscious state. J. Clin. Invest. 116: 2005-2011.
110. Brefel-Courbon, C. et al. 2007. Clinical and imaging evidence of zolpidem effect in hypoxic encephalopathy. Ann. Neurol. 62: 102-105.

111. SCHIFF, N.D. et al. 2007. Behavioral improvements with thalamic stimulation after severe traumatic brain injury. Nature 448: 600-603.

112. SCHIFF, N.D. et al. 2005. fMRI reveals large-scale network activation in minimally conscious patients. Neurology 64: $514-523$.

113. Schiff, N.D., F. Plum \& A.R. Rezai. 2002. Developing prosthetics to treat cognitive disabilities resulting from acquired brain injuries. Neurol. Res. 24: 116-124.

114. KATO, T. et al. 2007. Statistical image analysis of cerebral glucose metabolism in patients with cognitive impairment following diffuse traumatic brain injury. J. Neurotrauma 24: 919-926.

115. Whyte, J. et al. 2005. Predictors of outcome in prolonged posttraumatic disorders of consciousness and assessment of medication effects: A multicenter study. Arch. Phys. Med. Rehabil. 86: 453-462.

116. Schiff, N.D. \& J.P. Posner. 2007. Another "Awakenings." Ann. Neurol. 62: 5-7.

117. Grillner, S. et al. 2005. Mechanisms for selection of basic motor programs - roles for the striatum and pallidum. Trends Neurosci. 28: 364-370.

118. Chen, L. et al. 2004. Electrophysiological and behavioral effects of zolpidem in rat globus pallidus. Exp. Neurol. 186: $212-220$.

119. Thomas, S.A., A.E. Hudson, K.P. Purpura, N.D. SCHIFF. 2006. Corticothalamic synchronization organized around task-related behavioral events. Society for Neuroscience 36th Annual Meeting Abstract 138.16. 